\title{
DKOU 2010
}

\section{DKOU 2010}

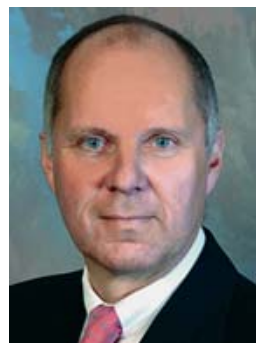

D. Frank

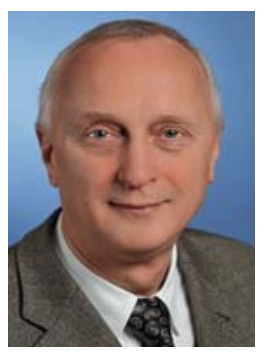

N. Südkamp

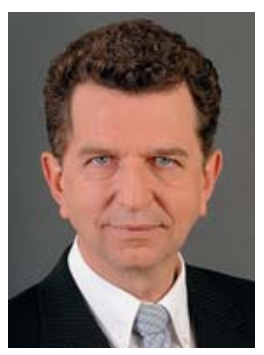

H. Mälzer

Bibliografie

DOI http://dx.doi.org/

10.1055/s-0030-1250363

Z Orthop Unfall 2010; 148: 519

(C) Georg Thieme Verlag KG

Stuttgart · New York .

ISSN 1864-6697
Der diesjährige Deutsche Kongress für Orthopädie und Unfallchirurgie findet vom 26.29.10.2010 wiederum in Berlin statt. Das Motto des Kongresses ist: Sicherheit, Zuverlässigkeit, Innovation.

Die bewährte Gliederung der Vorträge in Kurse, Tipps \& Tricks sowie Expertenrunden wurde wiederum übernommen. Die Kurse finden Mittwoch bis Freitag jeweils von 7:45 Uhr bis 9:15 Uhr statt und richten sich an Ärztinnen und Ärzte in Ausbildung. Die Rubrik Tipps \& Tricks ist für Kollegen im Facharztstatus zur Vermittlung praxisrelevanter Operationstechniken mit Darstellung von Fallstricken und Komplikationen gedacht. Für die niedergelassenen Kollegen ist die Vermittlung von wissenschaftlichen und betriebswirtschaftlichen Kenntnissen geplant. Die Expertenrunden richten sich an Ärztinnen und Ärzte in leitender Funktion für spezielle Fragestellungen.

Erstmalig wird dieses Jahr der „Specialty Day“ am Dienstag und Mittwochvormittag stattfinden. Die Sektionen und Arbeitskreise der DGOOC und der DGU haben ihre Vortragsblöcke in Eigenregie gestaltet.

Der Kongress wird in Fortführung der vorangegangenen Jahrestagungen weiter internationaler ausgerichtet. In diesem Jahr sind die Niederländer unser Partnerland und beteiligen sich aktiv mit einer Vielzahl von Vorträgen zu wissenschaftlichen Themen der Orthopädie und Unfallchirurgie und zur Berufspolitik. Es wurden weitere internationale Referenten aus den USA, Frankreich und China eingeladen. Der Saal 8 wird durchgängig mit englischsprachigen Themen gefüllt.

Entsprechend dem diesjährigen Motto unseres Kongresses erwarten wir von den über 800 eingeladenen Referenten ihre Position hinsichtlich der Zuverlässigkeit und Sicherheit der angewandten Diagnostik und Therapie. Die Patientensicherheit ist zunehmend im öffentlichen Fokus. Es wurden mehrere Sitzungen zu diesem Themenkomplex zusammengestellt. Ohne Innovation gibt es keinen Fortschritt, ob die Innovation immer mit besseren Ergebnissen verbunden ist, werden wir erfahren.

Mit der Deutschen Röntgengesellschaft wurde eine Zusammenarbeit vereinbart, sodass wir über die neuen wissenschaftlichen Ergebnisse sowie neue Techniken der Bildgebung informiert werden. In einer berufspolitischen Sitzung werden wir die Schnittstelle zwischen Radiologen und Teilgebietsradiologen beleuchten.
Die Bundeswehr wird in eigenen Vortragsblocks über ihre Erfahrungen im Auslandseinsatz sowie der Katastrophenmedizin berichten. Zum interprofessionellen Tag werden die OP-Pflege, die Physiotherapeuten sowie die Rettungssanitäter eingeladen.

Zur Eröffnungsveranstaltung hat Bundesgesundheitsminister Dr. Rösler zugesagt. Obwohl er mit seinen Plänen zur Zukunftssicherung des Gesundheitssystems auf heftige Kritik stößt, zeigt er eine bewundernswerte Standfestigkeit in diesem schwierigen Ressort. Zur Mittagsvorlesung am Mittwoch wurde der Regisseur und Buchautor Volker Schlöndorff eingeladen. Am Donnerstag wird Prof. Kirchhof, Vizepräsident des Bundesverfassungsgerichts, zur Freizügigkeit bei medizinischen Dienstleistungen in der EU und in Deutschland sprechen. Ein Thema, das sicherlich alle im Gesundheitswesen Tätigen interessieren dürfte. Prof. Harms ist sicherlich einer der herausragenden Wirbelsäulenchirurgen in Deutschland. Im Rahmen der Pauwels-Gedächtnisvorlesung am Freitag wird er über die Biomechanik und deren Bedeutung für die Therapie berichten.

Wir öffnen den Kongress für unseren Nachwuchs und die Jugend. Der Tag der Studierenden eröffnet interessierten Studenten die Möglichkeit, mit finanzieller Unterstützung den Kongress zu besuchen und von Tutoren begleitet zu werden. Den Kindern der Kongressteilnehmern bieten wir einen „begleiteten Tag“ mit Besuch des Kongresses, Hands-on und Berlinbesichtigung an - Meet your Parents. Und für die jüngsten ist die „Kinderinsel“ reserviert, eine ganztägige, professionelle Betreuung für alle Kinder bis zum 12. Lebensjahr.

Neben der Eröffnungsparty am 26.10.2010 bietet die Kongressparty am 28.10.2010 allen Teilnehmern die Möglichkeit der Begegnung und des Kennenlernens abseits der Wissenschaft.

Die Themen des Kongresses sind in diesem Jahr breit angelegt und in der Wahl der Referenten auf die aktuelle Situation im Gesundheitswesen und besonders der Orthopädie und Unfallchirurgie ausgerichtet. Die Kongresspräsidenten zusammen mit ihren Teams würden sich freuen, Sie auf dem Kongress begrüßen zu können.

Dr. med. Daniel Frank, Präsident DGOOC Prof. Dr. med. Norbert Südkamp, Präsident DGU Helmut Mälzer, Präsident BVOU 